\title{
Low-energy sector quantization of a massless scalar field outside a Reissner-Nordström black hole and static sources
}

\author{
J. Castiñeiras and G. E. A. Matsas \\ Instituto de Física Teórica, Universidade Estadual Paulista, Rua Pamplona 145, 01405-900, São Paulo, São Paulo, Brazil
}

(Received 22 February 2000; published 31 July 2000)

\begin{abstract}
We quantize the low-energy sector of a massless scalar field in Reissner-Nordstrom spacetime. This allows the analysis of processes involving soft scalar particles occurring outside charged black holes. In particular, we compute the response of a static scalar source interacting with Hawking radiation using the Unruh (and the Hartle-Hawking) vacuum. This response is compared with the one obtained when the source is uniformly accelerated in the usual vacuum of Minkowski spacetime with the same proper acceleration. We show that both responses are in general different in opposition to the result obtained when the Reissner-Nordström black hole is replaced by a Schwarzschild one. The conceptual relevance of this result is commented on.
\end{abstract}

PACS number(s): 04.70.Dy, 04.62.+v

\section{INTRODUCTION}

We study the canonical quantization of a massless scalar field outside a Reissner-Nordström black hole. This is not easy to fully accomplish mostly because the explicit form of the positive- and negative-energy modes is unknown in terms of the usual special functions. This has led many researchers to use numerical methods to analyze quantum field issues in this and in similar backgrounds (see, e.g., [1] and references therein). Here we follow the procedure developed in Ref. [2] to analytically quantize the low-energy sector of the scalar field in Reissner-Nordström spacetime. This allows the analytic investigation of processes involving soft particles such as, e.g., the synchrotron radiation emitted by scalar sources orbiting charged black holes [3].

We use our results to analyze the following conceptual issue. It was recently found [4] that the responses of (i) a static scalar source in the Schwarzschild spacetime with the Unruh vacuum and of (ii) a uniformly accelerated scalar source in Minkowski spacetime with the usual vacuum are equivalent provided that both sources have the same proper acceleration. It would be interesting to study, thus, whether or not this equivalence is preserved when the Schwarzschild black hole is supplied with some electric charge. Because (structureless) static sources can only interact with zeroenergy particles, we can use our low-energy quantization to answer this question accurately. Eventually we show that the presence of electric charge in the black hole breaks the above equivalence. This in conjunction with the fact that no equivalence is found in the Schwarzschild spacetime when the scalar field is replaced by the Maxwell one [5] suggests that the equivalence found in [4] is not valid, in general, for other spacetimes and quantum fields. Whether or not there is something deeper behind it remains an open question for us. We will adopt natural units $\hbar=c=G=k_{B}=1$ and signature $(+---)$.

The paper is organized as follows. In Sec. II we quantize the low-energy sector of the massless scalar field outside a Reissner-Nordström black hole. In Sec. III we compute the response of a static scalar source interacting with Hawking radiation using the Unruh (and the Hartle-Hawking) vacuum, and compare the result with the one obtained when the source is uniformly accelerated in Minkowski spacetime with the usual inertial vacuum. We present our final considerations in Sec. IV.

\section{QUANTIZATION OF A MASSLESS SCALAR FIELD OUTSIDE A CHARGED BLACK HOLE}

The line element of a Reissner-Nordström black hole with mass $M$ and electric charge $Q \leqslant M$ can be written as [6]

$$
d s^{2}=f(r) d t^{2}-f(r)^{-1} d r^{2}-r^{2}\left(d \theta^{2}+\sin ^{2} \theta d \varphi^{2}\right),
$$

where

$$
f(r) \equiv\left(1-r_{+} / r\right)\left(1-r_{-} / r\right)
$$

and $r_{ \pm} \equiv M \pm \sqrt{M^{2}-Q^{2}}$. Outside the outer event horizon, i.e., for $r>r_{+}$, we have a global timelike isometry generated by the Killing field $\partial_{t}$.

Let us now consider a free massless scalar field $\Phi\left(x^{\mu}\right)$ in this background described by the action

$$
S=\frac{1}{2} \int d^{4} x \sqrt{-g} \nabla^{\mu} \Phi \nabla_{\mu} \Phi,
$$

where $g \equiv \operatorname{det}\left\{g_{\mu \nu}\right\}$. In order to quantize the field, we look for a complete set of positive-energy solutions of the KleinGordon equation, $\square u_{\omega l m}=0$, in the form

$$
u_{\omega l m}=\sqrt{\frac{\omega}{\pi}} \frac{\psi_{\omega l}(r)}{r} Y_{l m}(\theta, \varphi) e^{-i \omega t}
$$

where $\omega \geqslant 0, l \geqslant 0$ and $m \in[-l, l]$ are the frequency and angular momentum quantum numbers. The factor $\sqrt{\omega / \pi}$ was inserted for later convenience and $Y_{l m}(\theta, \varphi)$ are the spherical harmonics. As a consequence $\psi_{\omega l}(r)$ must satisfy

$$
\left[-f(r) \frac{d}{d r}\left(f(r) \frac{d}{d r}\right)+V_{\mathrm{eff}}(r)\right] \psi_{\omega l}(r)=\omega^{2} \psi_{\omega l}(r),
$$

where the effective scattering potential $V_{\text {eff }}(r)$ is given by 


$$
V_{\mathrm{eff}}(r)=\left(1-\frac{2 M}{r}+\frac{Q^{2}}{r^{2}}\right)\left(\frac{2 M}{r^{3}}-\frac{2 Q^{2}}{r^{4}}+\frac{l(l+1)}{r^{2}}\right) .
$$

Note that Eq. (2.5) admits two sets of independent solutions which will be labeled by $\psi_{\omega l}^{\alpha}(r)$ with $\alpha=I, I I$. As a result, we can expand the scalar field $\Phi\left(x^{\mu}\right)$ in terms of annihilation $a_{\omega l m}^{\alpha}$ and creation $a_{\omega l m}^{\alpha \dagger}$ operators, as usual

$$
\Phi\left(x^{\mu}\right)=\sum_{\alpha=I, I I} \sum_{l=0}^{\infty} \sum_{m=-l}^{m=+l} \int_{0}^{+\infty} d \omega\left[u_{\omega l m}^{\alpha}\left(x^{\mu}\right) a_{\omega l m}^{\alpha}+\text { H.c. }\right],
$$

where $u_{\omega l m}^{\alpha}\left(x^{\mu}\right)$ are orthonormalized according to the KleinGordon inner product [7]:

$$
\begin{aligned}
& i \int_{\Sigma_{t}} d \Sigma n^{\mu}\left(u_{\omega l m}^{\alpha} * \nabla_{\mu} u_{\omega^{\prime} l^{\prime} m^{\prime}}^{\alpha^{\prime}}-\nabla_{\mu} u_{\omega l m}^{\alpha} * \cdot u_{\omega^{\prime} l^{\prime} m^{\prime}}^{\alpha^{\prime}}\right) \\
& \quad=\delta_{\alpha \alpha^{\prime}} \delta_{l l^{\prime}} \delta_{m m^{\prime}} \delta\left(\omega-\omega^{\prime}\right), \\
& i \int_{\Sigma_{t}} d \Sigma n^{\mu}\left(u_{\omega l m}^{\alpha} \nabla_{\mu} u_{\omega^{\prime} l^{\prime} m^{\prime}}^{\alpha^{\prime}}-\nabla_{\mu} u_{\omega l m}^{\alpha} \cdot u_{\omega^{\prime} l^{\prime} m^{\prime}}^{\alpha^{\prime}}\right) \\
& \quad=0 .
\end{aligned}
$$

Here $n^{\mu}$ is the future-pointing unit vector normal to the volume element of the Cauchy surface $\Sigma_{t}$. As a consequence, $a_{\omega l m}^{\alpha}$ and $a_{\omega l m}^{\alpha \dagger}$ satisfy simple commutation relations

$$
\left[a_{\omega l m}^{\alpha}, a_{\omega^{\prime} l^{\prime} m^{\prime}}^{\alpha^{\prime} \dagger}\right]=\delta_{\alpha \alpha^{\prime}} \delta_{l l^{\prime}} \delta_{m m^{\prime}} \delta\left(\omega-\omega^{\prime}\right)
$$

The Boulware vacuum $|0\rangle$ is defined by $a_{\omega l m}^{\alpha}|0\rangle=0$ for every $\alpha, \omega, l$, and $m[8]$.

\section{A. Small frequency modes}

The general solution of Eq. (2.5) in terms of special functions is not known. However, this can be found for small frequencies as follows. First let us rewrite Eq. (2.5) with $\omega$ $=0$ as

$$
\frac{d}{d z}\left[\left(1-z^{2}\right) \frac{d}{d z}\left[\psi_{\omega l}(y) / y\right]\right]+l(l+1)\left[\psi_{\omega l}(y) / y\right]=0,
$$

where we have defined $y \equiv r / 2 M, y_{ \pm} \equiv r_{ \pm} / 2 M$, and

$$
z \equiv \frac{2 y-1}{y_{+}-y_{-}}
$$

From the Legendre equation (2.11), we obtain the two independent solutions

$$
\begin{aligned}
& \psi_{\omega l}^{I}(y) \equiv C_{\omega}^{I} y Q_{l}[z(y)], \\
& \psi_{\omega l}^{I I}(y) \equiv C_{\omega}^{I I} y P_{l}[z(y)],
\end{aligned}
$$

where $Q_{l}(z)$ and $P_{l}(z)$ are the Legendre functions, and $C_{\omega}^{I}$ and $C_{\omega}^{I I}$ are normalization constants. In order to determine them, we shall analyze in more detail the solutions of Eq. (2.5) near the horizon and at infinity, which can be normalized for arbitrary $\omega$.

\section{B. Normal modes near the horizon and at infinity}

First let us note that by making the change of variables,

$$
y \rightarrow x=y+\frac{\left(y_{+}\right)^{2} \ln \left|y-y_{+}\right|-\left(y_{-}\right)^{2} \ln \left|y-y_{-}\right|}{y_{+}-y_{-}},
$$

Eq. (2.5) takes the form

$$
\left[-\frac{d^{2}}{d x^{2}}+4 M^{2} V_{\mathrm{eff}}[r(x)]\right] \psi_{\omega l}(x)=4 M^{2} \omega^{2} \psi_{\omega l}(x)
$$

It is convenient to write the two independent solutions of Eq. (2.16) such that $\psi_{\omega l}(x)$ and $\psi_{\omega l}^{\leftarrow}(x)$ are associated with purely incoming modes from the past white-hole horizon $\mathcal{H}^{-}$and from the past null infinity $\mathcal{J}^{-}$, respectively. These modes are orthogonal to each other with respect to the KleinGordon inner product (2.8). This can be seen by choosing $\Sigma_{t}=\mathcal{H}^{-} \cup \mathcal{J}^{-}$in Eq. (2.8) and recalling that $\psi_{\omega l}(x)$ and $\psi_{\omega l}^{\leftarrow}(x)$ vanish on $\mathcal{J}^{-}$and $\mathcal{H}^{-}$, respectively. Hence, by noting from Eq. (2.6) that close to $(x<0,|x| \gg 1)$ and far away from $(x \gg 1)$ the horizon, the scattering potential becomes $V_{\text {eff }}(r) \approx 0$ and $V_{\text {eff }}(r) \approx l(l+1) / r^{2}$, respectively, we write

$$
\psi_{\omega l}^{\overrightarrow{\omega l}}(x) \approx \begin{cases}A_{\omega l}\left(e^{2 i M \omega x}+\mathcal{R} \underset{\omega l}{\overrightarrow{ }} e^{-2 i M \omega x}\right) & (x<0,|x| \gg 1), \\ 2 i^{l+1} A_{\omega l} \mathcal{T}_{\omega l} M \omega x h_{l}^{(1)}(2 M \omega x) & (x \gg 1),\end{cases}
$$

and

$$
\psi_{\omega l}^{\leftarrow}(x) \approx \begin{cases}B_{\omega l} \mathcal{T}_{\omega l}^{\leftarrow} e^{-2 i M \omega x} & (x<0,|x| \gg 1), \\ B_{\omega l}\left[2(-i)^{l+1} M \omega x h_{l}^{(1)}(2 M \omega x)^{*}+2 i^{l+1} \mathcal{R}_{\omega l}^{\leftarrow} M \omega x h_{l}^{(1)}(2 M \omega x)\right] & (x \gg 1) .\end{cases}
$$


Here $h_{l}^{(1)}(2 M \omega x)$ are the spherical Hankel functions and $\left|\mathcal{R}_{\omega l}^{\leftarrow}\right|^{2},\left|\mathcal{R}_{\omega l}^{\vec{\omega}}\right|^{2}$ and $\left|\mathcal{T}_{\omega l}^{\leftarrow}\right|^{2},\left|\mathcal{T}_{\omega l}^{\vec{\omega}}\right|^{2}$ are the reflection and transmission coefficients, respectively, satisfying the usual probability conservation equations $\left|\mathcal{R}_{\omega l}\right|^{2}+\left|\mathcal{T}_{\omega l}\right|^{2}=1$ and $\left|\mathcal{R}_{\omega l}^{\leftarrow}\right|^{2}+\left|\mathcal{T}_{\omega l}^{\leftarrow}\right|^{2}=1$. Note that $h_{l}^{(1)}(x) \approx(-i)^{l+1} \exp (i x) / x$ for $|x| \gg 1$. The normalization constants $A_{\omega l}$ and $B_{\omega l}$ are obtained (up to an arbitrary phase) by letting normal modes (2.4) in the Klein-Gordon inner product (2.8) and using Eq.

(2.16) to transform the integral into a surface term:

$$
\begin{aligned}
& \frac{1}{\omega-\omega^{\prime}}\left[\psi_{\omega l}(x) \frac{d}{d x} \psi_{\omega^{\prime} l}^{*}(x)-\psi_{\omega^{\prime} l}^{*}(x) \frac{d}{d x} \psi_{\omega l}(x)\right]||_{x \rightarrow-\infty}^{x \rightarrow+\infty} \\
& \quad=\frac{2 \pi M}{\omega} \delta\left(\omega-\omega^{\prime}\right)
\end{aligned}
$$

By using the asymptotic solutions (2.17),(2.18) in Eq. (2.19), we obtain $A_{\omega l}=B_{\omega l}=(2 \omega)^{-1}$.

\section{Normalization constants}

Now we are able to determine the normalization constants $C_{\omega}^{I}$ and $C_{\omega}^{I I}$ by comparing Eqs. (2.13),(2.14) close and far away from the black hole with our normalized functions (2.17), (2.18) in the low-frequency regime $(2 M \omega x \ll 1)$. Let us begin by noticing that for $2 M \omega x \ll 1$, we have, near the horizon [see Eq. (2.17)],

$$
\psi_{\omega l}^{\overrightarrow{\omega l}}(x) \approx M x\left[\frac{\left(1+\mathcal{R}_{\omega l}^{\vec{\omega}}\right)}{2 M \omega x}+i\left(1-\mathcal{R}_{\omega l}^{\overrightarrow{\omega l}}\right)\right] \quad(x<0,|x| \gg 1) .
$$

In order that Eq. (2.20) have a good behavior in the lowfrequency regime we conclude that $\mathcal{R}_{\omega l}^{\rightarrow} \approx-1+\mathcal{O}(\omega)$. As a consequence, for $2 M \omega x \ll 1$ we obtain from Eq. (2.20) that

$$
\underset{\omega l}{\vec{\omega}}(x) \approx 2 i M x \quad(x<0,|x| \gg 1) .
$$

Now, we recall that in the low-frequency regime $\underset{\omega l}{\overrightarrow{ }}(x)$ is mostly reflected by the scattering potential back to the horizon and thus cannot be associated with $\psi_{\omega l}^{I I}(x)$ which grows asymptotically [see Eq. (2.14) and recall that $P_{l}(z) \sim z^{l}$ as $\left.z \gg 1\left(r \gg r_{+}\right)\right]$. This is not so for $\psi_{\omega l}^{I}(x)$ which decreases asymptotically and indeed fits $\psi_{\omega l}(x)$. This can be shown as follows. Let us first note that, for $z \approx 1\left(r \approx r_{+}\right)$,

$$
\begin{aligned}
Q_{l}(z) & \approx \frac{1}{2} \ln \left|\frac{z+1}{z-1}\right|-\sum_{k=1}^{l} \frac{1}{k} \\
& \approx \frac{\left[-x+y_{+}+\ln \left(y_{+}-y_{-}\right)\right]\left(y_{+}-y_{-}\right)}{2 y_{+}^{2}}-\sum_{k=1}^{l} \frac{1}{k},
\end{aligned}
$$

where we have used Eqs. (2.12) and (2.15). Thus, close to the horizon, we obtain from Eq. (2.13) that

$$
\psi_{\omega l}^{I}(x) \approx-C_{\omega}^{I} \frac{\left(y_{+}-y_{-}\right)}{2 y_{+}} x \quad(x<0,|x| \gg 1) .
$$

Comparing Eqs. (2.23) and (2.21) we find the normalization constant

$$
C_{\omega}^{I}=-4 i M y_{+} /\left(y_{+}-y_{-}\right) .
$$

Therefore, we write, from Eq. (2.13),

$$
\psi_{\omega l}^{I}(x)=\frac{-4 i M y_{+} y Q_{l}[z(y)]}{y_{+}-y_{-}},
$$

and from Eq. (2.4), we obtain the corresponding normalized low-frequency modes (up to an arbitrary phase):

$$
u_{\omega l m}^{I}\left(x^{\mu}\right)=\frac{2 y_{+} \omega^{1 / 2}}{\pi^{1 / 2}\left(y_{+}-y_{-}\right)} Q_{l}[z(x)] Y_{l m}(\theta, \varphi) e^{-i \omega t} .
$$

Now we fit $\psi_{\omega l}^{I}(x)$ and $\overrightarrow{\omega l}(x)$ asymptotically to determine the low-frequency transmission coefficient $\left|\mathcal{T}_{\omega l}\right|^{2}[$ see Eq. (2.17)]. For $x \gg 1$, Eq. (2.25) becomes

$$
\psi_{\omega l}^{I}(x) \approx \frac{-2 i M(l !)^{2} y_{+}\left(y_{+}-y_{-}\right)^{l} x^{-l}}{(2 l+1) !} \quad(2 M \omega x \ll 1),
$$

where we have used that, in this region,

$$
Q_{l}\left[2 y /\left(y_{+}-y_{-}\right)\right] \approx \frac{(l !)^{2}\left(y_{+}-y_{-}\right)^{l+1} y^{-l-1}}{2(2 l+1) !} .
$$

Now, from Eq. (2.17), we have in the low-frequency regime and for $x \gg 1$ that

$$
\psi_{\omega l}(x) \approx \frac{i^{l}(2 l) ! \mathcal{T}_{\omega l} x^{-l}}{2^{2 l+1} l ! M^{l} \omega^{l+1}} \quad(2 M \omega x \ll 1)
$$

where we have used that

$$
h_{l}^{(1)}(2 M \omega x)=j_{l}(2 M \omega x)+i n_{l}(2 M \omega x)
$$

and the fact that the spherical Bessel and Newman functions satisfy [see Eq. (11.156) of Ref. [9]]

$$
j_{l}(2 M \omega x) \approx \frac{2^{l} l !}{(2 l+1) !}(2 M \omega x)^{l}
$$

and

$$
n_{l}(2 M \omega x) \approx-\frac{(2 l) !}{2^{l} l !}(2 M \omega x)^{-(l+1)},
$$

respectively, for $2 M \omega x \ll 1$. Thus Eqs. (2.27) and (2.28) coincide provided that

$$
\mathcal{T}_{\omega l}^{\rightarrow}=\frac{2^{2 l+2}(-i)^{l+1} y_{+}\left(y_{+}-y_{-}\right)^{l}(l !)^{3}(M \omega)^{l+1}}{(2 l+1) !(2 l) !} .
$$

(Eventually this will be also used as a consistency check for our calculations.) 
Now, let us turn our attention to $\psi_{\omega l}^{\leftarrow}(x)$ which should be fitted with $\psi_{\omega l}^{I I}(x)$. Note that $\psi_{\omega l}^{I}(x)$ grows close to the horizon and so cannot be associated with low-frequency leftmoving modes which must be mostly reflected back to infinity by the scattering potential [see Eq. (2.13) and recall that $Q_{l}(z) \approx-\log |z-1|^{1 / 2}$ as $\left.z \approx 1\right]$. In order to fit $\psi_{\omega l}^{\leftarrow}(x)$ and $\psi_{\omega l}^{I I}(x)$ asymptotically, we must use Eqs. (2.29) and (2.30),(2.31) in Eq. (2.18) for $x \gg 1$. Moreover, it turns out that this compatibility is achieved if and only if $\mathcal{R} \overleftarrow{\omega l}$ $\approx(-1)^{l+1}$. As a result we obtain

$$
\psi_{\omega l}^{\leftarrow}(x) \approx \frac{2^{2 l+1}(-i)^{l+1} l ! \omega^{l}(M x)^{l+1}}{(2 l+1) !} \quad(x \gg 1)
$$

for $2 M \omega x \ll 1$. Now, we note that $P_{l}(z) \approx\left[(2 l) ! / 2^{l}(l !)^{2}\right] z^{l}$ for $z \gg 1$ [see Eqs. (8.837.2) and (8.339.2) of Ref. [10]]. Hence, using Eqs. (2.12) and (2.14), we find that

$$
\psi_{\omega l}^{I I}(x) \approx C_{\omega}^{I I} \frac{(2 l) ! y^{l+1}}{(l !)^{2}\left(y_{+}-y_{-}\right)^{l}} \quad(x \gg 1) .
$$

Comparing this equation with Eq. (2.33) and recalling that $x \approx y$ at infinity, we find the normalization constant

$$
C_{\omega}^{I I}=\frac{2^{2 l+1}(-i)^{l+1}(l !)^{3} M^{l+1}\left(y_{+}-y_{-}\right)^{l} \omega^{l}}{(2 l+1) !(2 l) !} .
$$

Therefore

$$
\psi_{\omega l}^{I I}(x)=\frac{2^{2 l+1}(-i)^{l+1}(l !)^{3} M^{l+1}\left(y_{+}-y_{-}\right)^{l} \omega^{l} y P_{l}[z(y)]}{(2 l+1) !(2 l) !}
$$

and the corresponding normalized small frequency modes are (up to an arbitrary phase)

$$
\begin{aligned}
u_{\omega l m}^{I I}\left(x^{\mu}\right)= & \frac{2^{2 l}(l !)^{3} M^{l}\left(y_{+}-y_{-}\right)^{l} \omega^{l+1 / 2}}{\pi^{1 / 2}(2 l+1) !(2 l) !} \\
& \times P_{l}[z(x)] Y_{l m}(\theta, \varphi) e^{-i \omega t} .
\end{aligned}
$$

It can be directly verified that by fitting Eq. (2.36) close to the horizon with Eq. (2.18) for $2 M \omega x \ll 1$, we obtain $\mathcal{T}_{\omega l}^{\leftarrow}$ $=\mathcal{T}_{\omega l}$ l [see Eq. (2.32)], as indeed required for consistency. Clearly this guaranties that $\left|\mathcal{R}_{\omega l}^{\leftarrow}\right|=\left|\mathcal{R}_{\omega l}\right|$. Note, however, that $\mathcal{R}_{\omega l}^{\leftarrow}$ and $\mathcal{R}_{\omega l} \vec{l}$ will in general differ by a phase (in contrast to $\mathcal{T}_{\omega l}^{\leftarrow}$ and $\left.\mathcal{T} \overrightarrow{\omega l}\right)$.

Equation (2.7) in conjunction with Eqs. (2.26) and (2.37) concludes our low-frequency sector quantization.

\section{RESPONSE OF A STATIC SCALAR SOURCE INTERACTING WITH HAWKING RADIATION}

Let us now compute the response of a static source to the Hawking radiation in the Reissner-Nordström spacetime. We will consider both Unruh and Hartle-Hawking vacua. Let us describe our pointlike scalar source lying at $\left(r_{0}, \theta_{0}, \varphi_{0}\right)$ by

$$
j\left(x^{\mu}\right)=\frac{q}{\sqrt{-h}} \delta\left(r-r_{0}\right) \delta\left(\theta-\theta_{0}\right) \delta\left(\varphi-\varphi_{0}\right),
$$

where $q$ is a small coupling constant and $h=-f^{-1} r^{4} \sin ^{2} \theta$ is the determinant of the spatial metric induced over the equal time hypersurface $\Sigma_{t}$. Note that Eq. (3.1) guarantees that

$$
\int_{\Sigma_{t}} d \Sigma j=q
$$

wherever the source lies. Let us now couple our source $j\left(x^{\mu}\right)$ to a massless scalar field $\Phi\left(x^{\mu}\right)$ as described by the interaction action

$$
S_{I}=\int d^{4} x \sqrt{-g} j \Phi
$$

The total source response, i.e., total particle emission and absorption probabilities per proper time associated with the source, is given by

$$
R \equiv \sum_{\alpha=I, I I} \sum_{l=0}^{\infty} \sum_{m=-l}^{l} \int_{0}^{+\infty} d \omega R_{\omega l m}^{\alpha}
$$

where

$$
R_{\omega l m}^{\alpha} \equiv \tau^{-1}\left\{\left.\left|\mathcal{A}_{\omega l m}^{\alpha} \mathrm{em}^{2}\left[1+n^{\alpha}(\omega)\right]+\right| \mathcal{A}_{\omega l m}^{\alpha}{ }^{\mathrm{abs}}\right|^{2} n^{\alpha}(\omega)\right\}
$$

and $\tau$ is the source's total proper time. (This is well defined since our source is pointlike.) Here $\mathcal{A}_{\omega l m}^{\alpha}{ }^{\mathrm{em}} \equiv\left\langle\alpha \omega l m\left|S_{I}\right| 0\right\rangle$ and $\mathcal{A}_{\omega l m}^{\alpha}$ abs $\equiv\left\langle 0\left|S_{I}\right| \alpha \omega l m\right\rangle$ are the emission and absorption amplitudes, respectively, of Boulware states $|\alpha \omega l m\rangle$, at the tree level. Moreover,

$$
n_{U}^{\alpha}(\omega) \equiv \begin{cases}\left(e^{\omega \beta}-1\right)^{-1} & \text { for } \alpha=I, \\ 0 & \text { for } \alpha=I I,\end{cases}
$$

and

$$
n_{H H}^{\alpha}(\omega) \equiv \begin{cases}\left(e^{\omega \beta}-1\right)^{-1} & \text { for } \alpha=I, \\ \left(e^{\omega \beta}-1\right)^{-1} & \text { for } \alpha=I I,\end{cases}
$$

for the Unruh and Hartle-Hawking vacua, respectively, with

$$
\beta^{-1}=\frac{y_{+}-y_{-}}{8 \pi M y_{+}^{2}} .
$$

We recall that the Unruh vacuum is characterized by a thermal flux leaving $\mathcal{H}^{-}$with Hawking temperature $\beta^{-1}$ at infinity given by Eq. (3.8) while the Hartle-Hawking vacuum has in addition a thermal flux coming from $\mathcal{J}^{-}$characterized by the same temperature at infinity [11].

Let us note that because structureless static sources (3.1) can only interact with zero-energy modes, the total response of this source in the Boulware vacuum vanishes. This is not so, however, in the presence of a background thermal bath since the absorption and (stimulated) emission rates render it nonzero. In order to deal with zero-energy modes, we need a 
"regulator" to avoid the appearance of intermediate indefinite results. (For a more comprehensive discussion on the interaction of static sources with zero-energy modes, see Ref. [12].) For this purpose we let the coupling constant $q$ smoothly oscillate with frequency $\omega_{0}$, writing Eq. (3.1) in the form

$$
j_{\omega_{0}}\left(x^{\mu}\right)=\frac{q_{\omega_{0}}}{\sqrt{-h}} \delta\left(r-r_{0}\right) \delta\left(\theta-\theta_{0}\right) \delta\left(\varphi-\varphi_{0}\right)
$$

where $q_{\omega_{0}} \equiv \sqrt{2} q \cos \left(\omega_{0} t\right)$ and taking the limit $\omega_{0} \rightarrow 0$ at the end. The factor $\sqrt{2}$ has been introduced to guarantee that the time average $\left\langle\left|q_{\omega_{0}}(t)\right|^{2}\right\rangle_{t}=q^{2}$ since at the tree level the absorption and emission rates are functions of $q^{2}$. By using Eqs. (3.9) and (2.7) in Eq. (3.3) we obtain the absorption amplitude

$$
\begin{aligned}
\mathcal{A}_{\omega l m}^{\alpha{ }^{\mathrm{abs}}=} & q \sqrt{2 \pi \omega_{0}}\left(\psi_{\omega_{0} l}^{\alpha}\left(r_{0}\right) / r_{0}\right) f^{1 / 2}\left(r_{0}\right) \\
& \times Y_{l m}\left(\theta_{0}, \varphi_{0}\right) \delta\left(\omega-\omega_{0}\right),
\end{aligned}
$$

and we recall that $\left|\mathcal{A}_{\omega l m}^{\alpha} \mathrm{em}\right|=\mid \mathcal{A}_{\omega l m}^{\alpha}$ abs $\mid$. By letting Eq. (3.10) into Eq. (3.5) we obtain

$$
\begin{aligned}
R_{\omega l m}^{\alpha}= & q^{2} \omega_{0}\left[\left|\psi_{\omega_{0} l}^{\alpha}\left(r_{0}\right)\right|^{2} / r_{0}^{2}\right] f^{1 / 2}\left(r_{0}\right) \\
& \times\left|Y_{l m}\left(\theta_{0}, \varphi_{0}\right)\right|^{2}\left[1+2 n^{\alpha}\left(\omega_{0}\right)\right] \delta\left(\omega-\omega_{0}\right),
\end{aligned}
$$

where it was used that the source's total proper time is $\tau$ $=2 \pi f^{1 / 2}\left(r_{0}\right) \lim _{\omega \rightarrow 0} \delta(\omega)$. [Here $f^{1 / 2}\left(r_{0}\right)$ is the gravitational redshift factor.]

Let us first consider the Unruh vacuum. By using Eqs. (2.25), (3.6), and (3.11) in Eq. (3.4) and making $\omega_{0} \rightarrow 0$ at the end, we compute the total response

$$
R_{U}=\frac{q^{2} a\left(M-Q^{2} / r_{+}\right)}{4 \pi^{2}\left(M-Q^{2} / r_{0}\right)}
$$

[note that modes $u_{\omega l m}^{I I}(x)$ do not give any contribution here], where

$$
a=\frac{f^{-1 / 2}\left(r_{0}\right)}{2} \frac{d f\left(r_{0}\right)}{d r_{0}}
$$

is the source's proper acceleration and we have used

$$
\sum_{m=-l}^{l}\left|Y_{l m}\left(\theta_{0}, \varphi_{0}\right)\right|^{2}=\frac{2 l+1}{4 \pi}
$$

and [4]

$$
\sum_{l=0}^{\infty}\left|Q_{l}(s)\right|^{2}(2 l+1)=\frac{1}{s^{2}-1} .
$$

Next we compare Eq. (3.12) with

$$
R_{M}=\frac{q^{2} a}{4 \pi^{2}}
$$

which is the response associated with our scalar source when it is uniformly accelerated in the usual vacuum of Minkowski spacetime with proper acceleration $a$. We note that although Eqs. (3.12) and (3.15) coincide when $Q=0$, as found in Ref. [4], they do not for $Q \neq 0$. As a result, the presence of electric charge inside the black hole breaks the response equivalence.

We note that the equality between Eqs. (3.12) and (3.15) is recovered when $r_{0} \approx r_{+}$. Hence, close to the horizon, a static source in the Unruh vacuum responds as if it were static in the Rindler wedge (i.e., uniformly accelerated in Minkowski spacetime) with the usual inertial vacuum provided that both sources have the same proper acceleration. Moreover, Eq. (3.12) can be written in this region in terms of the proper temperature [13] $\beta_{0}^{-1}=\beta^{-1} / \sqrt{f\left(r_{0}\right)}$ on the source's location as

$$
R_{U} \approx \frac{q^{2}}{2 \pi \beta_{0}}
$$

Equation (3.16) coincides with the response associated with our source when it is at rest in Minkowski spacetime with a background thermal bath characterized by a temperature $\beta_{0}^{-1}$. This result is not surprising because close to the horizon the scattering potential vanishes and the zero-energy modes leaving $\mathcal{H}^{-}$are completely reflected back towards the horizon.

Now let us turn our attention to the Hartle-Hawking vacuum. An analogous calculation leads us to the following response:

$$
R_{H H}=\frac{q^{2} a\left(M-Q^{2} / r_{+}\right)}{4 \pi^{2}\left(M-Q^{2} / r_{0}\right)}+\frac{q^{2}\left(M-Q^{2} / r_{+}\right)\left(M-Q^{2} / r_{0}\right)}{4 \pi^{2} r_{+}^{2} r_{0}^{2} a} \text {, }
$$

where we have used that $P_{0}\left[z\left(r_{0}\right)\right]=1$ and $Y_{00}=1 / \sqrt{4 \pi}$. [Note that, in this case, only $l=0$ contributes in Eq. (3.4).] The first term on the right-hand side of Eq. (3.17) is identical to the one obtained with the Unruh vacuum and is associated with the thermal flux leaving $\mathcal{H}^{-}$. The second term is associated with the thermal flux coming from $\mathcal{J}^{-}$. As a consistency check we note that for $r \rightarrow r_{+}$, we obtain $R_{H H}=R_{U}$. This should be so because close to the horizon, zero-energy particles coming from $\mathcal{J}^{-}$cannot overpass the scattering barrier. Consequently, in this limit, the second term on the right-hand side of Eq. (3.17) must vanish. Now, when the source is far away from the hole, the second term on the right-hand side of Eq. (3.17) dominates because zero-energy particles leaving $\mathcal{J}^{-}$are not able to reach the asymptotic region. Moreover, in this region, Eq. (3.17) can be rewritten in the form

$$
R_{H H} \approx \frac{q^{2}}{2 \pi \beta}
$$


Hence, far away from the hole, the source behaves as if it were in Minkowski spacetime immersed in a thermal bath with temperature $\beta^{-1}$, as expected.

\section{DISCUSSIONS}

We have quantized the low-energy sector of a massless scalar field in Reissner-Nordström spacetime. The results obtained were used to analyze the response of a static source interacting with Hawking radiation using the Unruh and Hartle-Hawking vacua. We have shown that, in general, static sources outside charged black holes (with the Unruh vacuum) do not behave similarly to uniformly accelerated sources in Minkowski spacetime (with the usual inertial vacuum) as previously found for neutral black holes [4]. This in conjunction with the fact that no equivalence is found in the Schwarzschild spacetime when the scalar field is replaced by the Maxwell one [5] shows that the equivalence found in [4] is not valid, in general, for other spacetimes and quantum fields. Whether or not there is something deeper behind it remains an open question for us. We have also verified that close to and far away from the horizon our source behaves as if it were at rest in a thermal bath in Minkowski spacetime with proper temperature associated with the Unruh and Hartle-Hawking vacua, respectively. The low-energy quantization presented here can be used to analyze other processes occurring outside charged black holes.

\section{ACKNOWLEDGMENTS}

We are thankful to L.C.B. Crispino for reading the manuscript. J.C. and G.M. would like to acknowledge full and partial support from the Fundação de Amparo à Pesquisa do Estado de São Paulo (FAPESP) and Conselho Nacional de Desenvolvimento Científico e Tecnológico (CNPq), respectively.
[1] P. R. Anderson, W. A. Hiscock, and D. A. Samuel, Phys. Rev. D 51, 4337 (1995).

[2] A. Higuchi, G. E. A. Matsas, and D. Sudarsky, Phys. Rev. D 58, 104021 (1998).

[3] L. C. B. Crispino, A. Higuchi, and G. E. A. Matsas, Class. Quantum Grav. 17, 19 (2000).

[4] A. Higuchi, G. E. A. Matsas, and D. Sudarsky, Phys. Rev. D 56, R6071 (1997).

[5] L. C. B. Crispino, A. Higuchi, and G. E. A. Matsas, Phys. Rev. D 58, 084027 (1998).

[6] R. W. Wald, General Relativity (University of Chicago Press, Chicago, 1984).

[7] N. D. Birrell and P. C. W. Davies, Quantum Field Theory in Curved Space (Cambridge University Press, Cambridge, England, 1982); S. A. Fulling, Aspects of Quantum Field Theory in Curved Space-Time (Cambridge University Press, Cam- bridge, England, 1989).

[8] D. G. Boulware, Phys. Rev. D 11, 1404 (1975); 12, 350 (1975).

[9] G. B. Arfken and H. J. Weber, Mathematical Method for Physicists (Academic, New York, 1995).

[10] I. S. Gradsthteyn and I. M. Ryzhik, Table on Integrals, Series and Products (Academic, New York, 1980).

[11] S. W. Hawking, Nature (London) 248, 30 (1974); Commun. Math. Phys. 43, 199 (1975); W. G. Unruh, Phys. Rev. D 14, 870 (1976); J. B. Hartle and S. W. Hawking, ibid. 13, 2188 (1976).

[12] A. Higuchi, G. E. A. Matsas, and D. Sudarsky, Phys. Rev. D 45, R3308 (1992); 46, 3450 (1992).

[13] R. C. Tolman, Relativity, Thermodynamics and Cosmology (Clarendon, Oxford, 1934). 